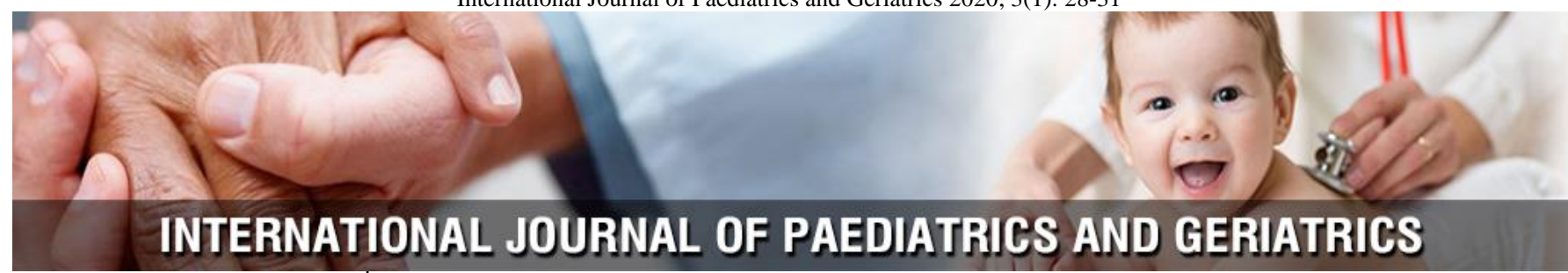

P-ISSN: 2664-3685

E-ISSN: 2664-3693

www.paediatricjournal.com

IJPG 2020; 3(1): 28-31

Received: 16-11-2019

Accepted: 20-12-2019

\section{Bhavani KC}

Resident, Department of Pediatrics, NIMS University, Jaipur, Rajasthan, India

\section{Virendra Kumar Gupta} Associate Professor, Department of Pediatrics, NIMS University, Jaipur, Rajasthan, India

Bhagwan Sahai Natani Associate Professor, Department of Pediatrics, NIMS University, Jaipur, Rajasthan, India

\section{Rupesh Masand}

Professor, Department of Pediatrics, NIMS University, Jaipur, Rajasthan, India

Chaman Ram Verma Professor, Department of Pediatrics, NIMS University, Jaipur, Rajasthan, India
Corresponding Author: Bhagwan Sahai Natani Associate Professor, Department of Pediatrics, NIMS University, Jaipur, Rajasthan, India

\section{Comparative analysis of serum vitamin d level in under five aged children with acute lower respiratory tract infection: A case-control study}

\author{
Bhavani KC, Virendra Kumar Gupta, Bhagwan Sahai Natani, Rupesh \\ Masand and Chaman Ram Verma
}

DOI: https://doi.org/10.33545/26643685.2020.v3.i1a.56

\begin{abstract}
Background: Vitamin D has been associated with the immune functions acting at the cell level and being involved with induction of different cells of immune system. It plays a major role in lung growth $\&$ function, which prevents infections of respiratory tract.

Methods: Hospital based prospective, observational, case control study was conducted in the Department of Pediatrics National Institute of medical Science and research, Jaipur, Rajasthan, from January 2017 to june 2018. A total of 250 children aged 1 to 5 years in each group with \& without ALRI, who fulfilled the inclusion criteria were included and Vitamin D levels were measured.

Results: In 250 cases mean age were $3.190 \pm 1.24,55.2 \%$ were males and $44.8 \%$ females. $60 \%$ of cases and $54 \%$ of control were from rural areas. Among cases $17.6 \%$ had insufficient and $64.4 \%$ had deficient vitamin D status whereas in control group $64.4 \%$ had normal, $22.8 \%$ insufficient and only $12.8 \%$ had deficiency of vitamin D. Highly significant p-value was obtained. $56.4 \%$ of cases had low serum calcium levels. Mean vitamin D levels was $19.9 \pm 11.46$ in cases and 33.21 \pm 12.30 in controls. Mean serum vitamin D of 16.9 in urban and 19 in rural area in cases whereas 30.6 and 35.3 in urban and rural areas respectively in control group.

Conclusions: We found a strong correlation of vitamin D deficiency with ALRI. Empirical supplementation of vitamin D can prevent the incidence of these infections. We can add Vitamin D in different public health programs.
\end{abstract}

Keywords: Respiratory tract infections, vitamin D level, serum Ca level, ALRI, supplementation

\section{Introduction}

Respiratory infections especially under the age of 5 years has been a leading cause of childhood morbidity and mortality in both developed as well as developing countries ${ }^{[1]}$. Worldwide acute lower respiratory infections (ALRI) are the major cause of death in children with 12 million hospitalizations globally with 1-2 million under-five deaths ${ }^{[2,3]}$.

Unfortunately, the burden and etiology of acute respiratory infection (ARI) including ALRI in India has not been well studied. In India, out of total deaths $17 \%$ were due to pneumonia in children less than 5 years old ${ }^{[4]}$. Study done in 2013 in India, the cost of an ALRIassociated hospitalization was high and estimated around $34 \%$ of annual per capita income ${ }^{[5]}$. Vitamin D is an essential fat soluble vitamin required for optimal health. Vitamin D2, or ergocalciferol, and vitamin D3, or cholecalciferol are the two major forms ${ }^{[6]}$. Vitamin D also known as the "sunshine vitamin," is a prohormone with lots of functions in human body ${ }^{[7]}$.

Vitamin D deficiency has been associated with dysregulation of the immune systems acting at the cell level and being involved with induction of different cells of immune system to fight against foreign attacking agents example being respiratory syncytial virus. It also helps in memory which prevents recurrent infections. Levels of 25-hydroxy Vitamin D and serum calcium are measured to assess the adequacy of Vitamin D stores in Ones body ${ }^{[8]}$. In our study we have tried to find out the vitamin D status of under-five age group admitted with a diagnosis of various respiratory illnesses like bronchiolitis, pneumonia, very severe pneumonia and other illness like bronchitis, influenza etc. This was compared with healthy children of the same age group.

In view of this we conducted the study with the aim to find out the relationship between vitamin D deficiency and ALRI in the age group one to five years. 


\section{Role of vitamin D in the mucosal immune response and Reduction of ALRI}

Figure 1 shows that vitamin D plays a role in mucosal immunity in view of increase innate immune function with different barrier and adaptive immune functions.

\section{Methods}

This was a hospital based prospective, observational, case control study was conducted in the Department of Pediatrics National Institute of medical Science and research, Jaipur, Rajasthan, from 1st January 2017 to 30th june 2018.

A total of 250 children aged between 1 to 5 years of either sex in each group with \& without acute lower respiratory tract infection who fulfilled the inclusion and exclusion criteria were included in the study after taking informed and written consentfrom parents or legal guardians. Ethical clearance was taken from institutional ethical clearance committee.

\section{Inclusion criteria}

- Children aged between 1 to 5 years of either sex were taken into the study.

- Children with clinical diagnosis of acute lower respiratory tract infections diagnosed according to WHO guidelines were taken as cases. (ARI Control Program)

\section{Exclusion criteria}

- Children less than one year and more than 5 Years of age.

- Children who received Vitamin D or calcium supplementation within last four weeks before admission.

- Children with

- Upper respiratory tract infections like otitis media \& pharyngitis.

- Bronchial asthma, Pulmonary tuberculosis, Lung Abscess, Concurrent empyema thoracis or illness severe enough to require ventilation.

- Clinical evidence of any heart disease, renal or hepatic insufficiency, known hypercalcemia or allergy to vitamin $\mathrm{D}$

- Metabolic disorder

- Protein Energy Malnutrition (weight for height less than 70\%; and/or height for age $<85 \%$, or presence of edema as per WHO classification of undernutrition)

\section{Methodology}

After taking detailed careful history from parents and relevant examination children aged one to five years having acute lower respiratory tract infection, diagnosed according WHO criteria were taken as cases and $2 \mathrm{ml}$ of venous blood taken in plane vial with all aseptic precautions. Serum levels of 25 hydroxy cholecalciferols were measured along with complete blood count, serum calcium and others as per requirements. X ray of chest was done. Age controlled 250 healthy children were taken as controls and $2 \mathrm{ml}$ of venous blood was drawn to measure 25 hydroxy cholecalciferols levels. Vitamin D levels were measured by using Fluorescence Immuno Assay- ELISA method. Deficiency of vitamin D was categorized on the basis of US Endocrine Society clinical practice guideline $1^{[9]}$.
Vitamin D Level cutoff values Categorized ${ }^{[9]}$.

$\begin{array}{lll}\text { Deficiency } & : & <20 \mathrm{ng} / \mathrm{ml} \\ \text { Insufficient } & : & 21 \text { to } 29 \mathrm{ng} / \mathrm{ml} \\ \text { Normal } & : & \geq 30 \mathrm{ng} / \mathrm{ml} .\end{array}$

\section{Statistical analysis}

Socio-economic and demographic variables were compared according to vitamin D status using ANOVA for continuous variables and chi square test of independence for categorical variables. Multinomial logistic regression was used to estimate the odds of being vitamin $\mathrm{D}$ deficient or insufficient with the reference being vitamin D sufficient children. A probability of less than 0.05 was considered a statistically significant association. All analysis was conducted using SPSS- version 22.

\section{Results}

A total 250 children were enrolled as cases with LRTI and 250 healthy controls one to five year of age. Mean age for the cases was $3.190 \pm 1.24$. $55.2 \%$ were males and $44.8 \%$ females. $60 \%$ of cases and $54 \%$ of control were from rural areas since our hospital caters to rural population of nearby villages.

Among cases $17.6 \%$ had insufficient and $64.4 \%$ had deficient vitamin D status where as in control group $64.4 \%$ had normal vitamin $\mathrm{D}, 22.8 \%$ had insufficient and only $12.8 \%$ had deficiency of vitamin D. Highly significant $p$ value was obtained showing strong association between lower respiratory tract illness and vitamin D deficiency [Table-1]. $56.4 \%$ of cases had low serum calcium levels showing strong association between lower respiratory tract illness and serum calcium levels [Table-2].

Mean vitamin D levels was $19.9 \pm 11.46$ in cases and $33.21 \pm 12.30$ in controls [figure-2]. Urban population had lower values of vitamin D in both the groups. Mean serum vitamin D of 16.9 in urban and 19 in rural area in cases group. In control group it was 30.6 in urban and 35.3 in rural area.

\section{Discussion}

Acute lower respiratory tract infection (ALRI) is a major cause of child mortality, annually causing approximately 1.8 million deaths of under five children ${ }^{[10]}$.

Salimpour found a link between pneumonia and vitamin D. He found $43 \%$ of rachitic children also had history of ALRI [11]. Wayse et al. studied ALRIs in children admitted and found a link between sub-clinical vitamin D deficiency, non-exclusive breastfeeding, and increased risk of severe ALRIs ${ }^{[12]}$. A meta-analysis of RCTs showed that vitamin D supplementation in children significantly reduced the risk of contracting RTIs (OR, 0.58; 95\% CI, 0.41-0.8) ${ }^{[13]}$.

As shown in table we found a strong association between Acute lower respiratory tract illness and vitamin D deficiency. This was found comparable to Study done by chandrashekar et al. ${ }^{[14]}$ where he found a significant correlation between lower levels of vitamin D and LRTI. Out of 59 cases with LRTI $50(84.7 \%)$ had Vitamin D deficiency, $6(10.1 \%)$ had insufficiency and only $3(5.08 \%)$ had normal Vitamin $\mathrm{D}$ values which was found to be statistically significant. Children with pneumonia were 43 $(72.88 \%)$ out of 59, among them 40 (93\%) had deficiency of Vitamin D. Authors also found that $13(22.03 \%)$ members were had recurrent LRTI out of which $84.6 \%$ had vitamin D deficiency. 
A similar cohort study was done in the Affiliated Children's Hospital of Capital Institute of Pediatrics, Beijing, China. This study investigated the effect of vitamin D status on the susceptibility and severity of viral pneumonia in children. They took 236 children aged from 1 month to 14 years having viral pneumonia, and 271 gender and age matched healthy children and compared the vitamin D status. The median serum $25(\mathrm{OH}) \mathrm{D}$ level in these children was 23.7 (IQR $17.5-30.6) \mathrm{ng} / \mathrm{mL}^{[15]}$. In our study as shown in figure2 we found mean vitamin D levels as 19.9 in cases and it was found comparatively much lower than in control group with a mean of 33.21. Their study was comparable to our study. In their study the median serum $25(\mathrm{OH}) \mathrm{D}$ level in the viral pneumonia group was much lower than in the control group 19.6 versus $26.6(P<0.001)$. Vitamin D deficiency $(32.2 \%$ versus $19.5 \%)$ and severe deficiency $(19.1 \%$ versus $0.4 \%$ ) in the viral pneumonia group was higher than control group.

Table 1: Comparison of vitamin D status in ALRI cases and healthy control

\begin{tabular}{|c|c|c|c|c|}
\hline & \multicolumn{2}{|c|}{ Cases } & \multicolumn{2}{c|}{ Control } \\
\hline Vitamin D & No. & \% & No. & \% \\
\hline Normal $(\geq 30 \mathrm{ng} / \mathrm{ml})$ & 45 & 18.0 & 161 & 64.4 \\
\hline Insufficient $(21-29 \mathrm{ng} / \mathrm{ml})$ & 44 & 17.6 & 57 & 22.8 \\
\hline Deficient $(\leq 20 \mathrm{ng} / \mathrm{ml})$ & 161 & 64.4 & 32 & 12.8 \\
\hline Total & 250 & 100 & 250 & 100 \\
\hline Chi-square Value & \multicolumn{4}{|c|}{153.21} \\
\hline P-Value & \multicolumn{4}{|c|}{00001} \\
\hline
\end{tabular}

Table 2: Comparison of serum calcium level in ALRI cases and healthy control

\begin{tabular}{|c|c|c|c|c|}
\hline & \multicolumn{2}{|c|}{ Cases } & \multicolumn{2}{c|}{ Control } \\
\hline Serum Ca & No. & \% & No. & \% \\
\hline Low $(\leq 8.4 \mathrm{mg} / \mathrm{dl})$ & 141 & 56.4 & 62 & 24.8 \\
\hline Normal $(8.5-10.3 \mathrm{mg} / \mathrm{dl})$ & 107 & 42.8 & 183 & 73.2 \\
\hline High $(\geq 10.4 \mathrm{mg} / \mathrm{dl})$ & 2 & 0.8 & 5 & 2 \\
\hline Total & 250 & 100 & 250 & 100 \\
\hline Chi-square Value & \multicolumn{4}{|c|}{52.70} \\
\hline P-Value & \multicolumn{4}{|c}{00001} \\
\hline
\end{tabular}

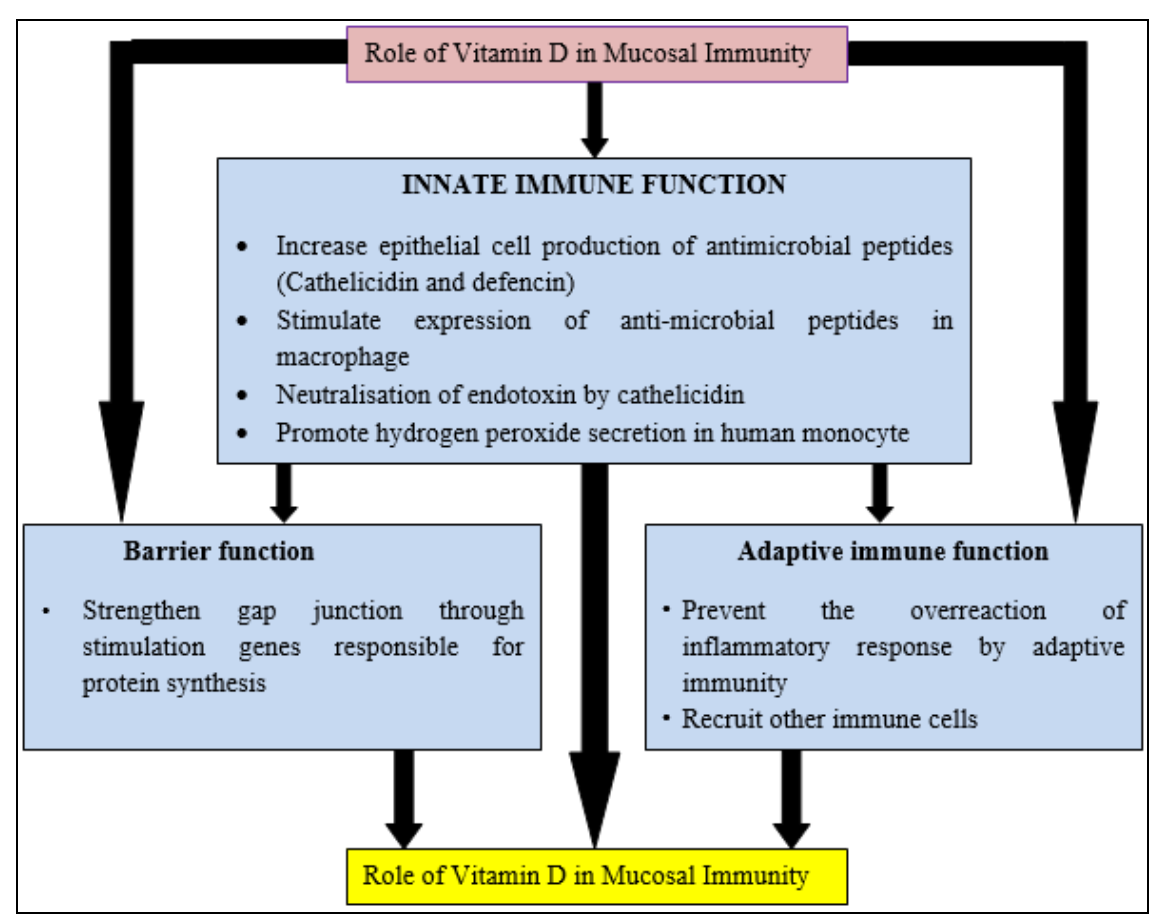

Fig 1: Role of vitamin D in the mucosal immune response and reduction of ARI

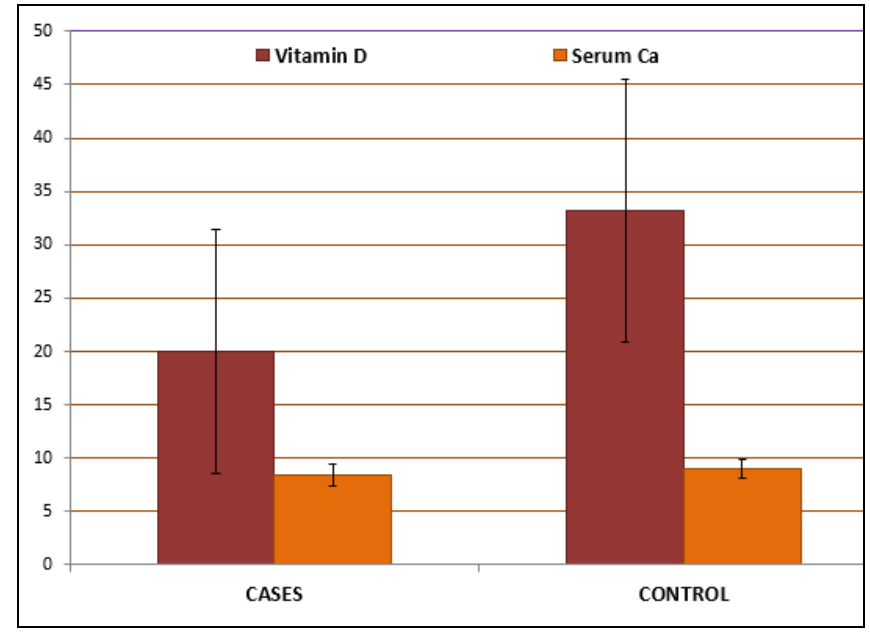

Fig 2: Mean vitamin D \& Serum Ca level

\section{Conclusion}

The basic question 'why illness occurs' should be answered. Acute lower respiratory tract illness is mainly infectious in nature with associated risk factors. There have been many studies done in the past. In this study we have tried to find out the relationship of vitamin $\mathrm{D}$ serum levels with that acute respiratory tract illness of children under 5 years of age. With the use of simple measures it will not only reduce the morbidity and mortality of young children but will also reduce the economic burden of any country. This is especially true for middle and lower income countries like India. Once it is proven that deficiency or insufficiency of vitamin $\mathrm{D}$ either acts as one of the causal factors or associated with increasing the severity of acute lower respiratory tract illness, then appropriate measures can be taken.

We found a strong correlation of vitamin D deficiency with 
acute lower respiratory tract illness. Although we need more studies to prove the relationship, also we need studies to determine whether supplementation of vitamin D actually reduces the illness. Whether empirical supplementation of vitamin D at various health centers prevent the incidence of these infections.

In a country like ours many problems have been tackled in the past at the national level by running various programmes. Vitamin $\mathrm{D}$ which can be given as a supplement at the grass root level in primary health centers can play a major role in improving the health of our country's children.

\section{References}

1. Krishnan A, Kumar R, Broor S et al. Epidemiology of viral acute lower respiratory infections in a communitybased cohort of rural north Indian children. J Glob Health. 2019; 9(1):010433. doi:10.7189/jogh.09.010433

2. Liu L, Oza S, Hogan D, Perin J, Rudan I, Lawn JE et al. Global, regional, and national causes of child mortality in 2000-13, with projections to inform post2015 priorities: an updated systematic analysis. Lancet. 2015; 385:430-40. Medline:25280870 doi:10.1016/S0140-6736(14):61698-6

3. Nair H, Simões EAF, Rudan I, Gessner BD, AzzizBaumgartner E, Zhang JSF et al. Global and regional burden of hospital admissions for severe acute lower respiratory infections in young children in 2010: a systematic analysis. Lancet. 2013; 381:1380-90. Medline:23369797 doi:10.1016/S0140-6736(12)619011.

4. Registrar General of India. Causes of Death in India 2010-2013. New Delhi. 2016. Available: http://www.censusindia.gov.in/2011-

Common/Sample_Registration_System.html Accessed: 9 April 2019.

5. Peasah SK, Purakayastha DR, Koul PA, Dawood FS, Saha S, Amarchand $\mathrm{R}$ et al. The cost of acute respiratory infectionsin Northern India: a multi-site study. BMC Public Health. 2015; 15:330. Medline:25880910 doi:10.1186/s12889-015-1685-6.

6. Norman AW, Bouillon R, Whiting SJ, Vieth R, Lips P. 13th Workshop consensus for vitamin $\mathrm{D}$ nutritional guidelines. J Steroid Biochem Mol Biol. 2007; 103:2

7. Holick MF. Sunlight and vitamin D for bone health and prevention of autoimmune diseases, cancers, and cardiovascular disease. Am J Clin Nutr. 2004; 80(6):1678S-88.

8. Cantorna, Margherita T. Mechanisms underlying the effect of vitamin D on the immune system." The Proceedings of the Nutrition Society. 2010; 69(3):2869. doi:10.1017/S0029665110001722

9. Holick MF, Binkley NC, Bischoff-Ferrari HA et al. Evaluation, treatment, and prevention of vitamin D deficiency: an Endocrine Society clinical practice guideline. J Clin Endocrinol Metab. 2011; 96:19111930.

10. Esposito S, Rigante D, Principi N. Do children's upper respiratory tract infections benefit from probiotics? BMC Infect Dis. 2014; 14:194.

11. Salimpour R. Rickets in Tehran. Arch Dis Child. 1975; 50:63-5.

12. Wayse V, Yousafzai A, Mogale K et al. Association of subclinical vitamin D deficiency with severe acute lower respiratory infection in Indian children under $5 \mathrm{y}$. Eur J Clin Nutr. 2004; 58:563-567.

13. Charan J, Goyal JP, Saxena D, Yadav PJ. Vitamin D for prevention of respiratory tract infections: a systematic review and meta-analysis. Pharmacol Pharmacother. 2012; 3:300-3.

14. Chandrashekhara, Pampana S. Prevalence of vitamin D deficiency in children with lower respiratory tract infection. Int J Contemp Pediatr. 2019; 6:1041-5.

15. Lin-Ying Guo, Wei Li, Xian-Fen Cheng, Hong-Ri Li, Chun-Rong Sun, Jing Guo et al. ediatric Allergy, Immunology, and Pulmonology, 2017. ahead of printhttp://doi.org/10.1089/ped.2017.0750 